\title{
EXPERIMENTAL RESULTS ON PBC OF A DOUBLY-FED INDUCTION GENERATOR INTERCONNECTED WITH AN INDUCTION MOTOR
}

\author{
M. Becherif ${ }^{*}$ E. Mendes ${ }^{* *}$ R. Ortega ${ }^{* * *}$ \\ J. Riera ${ }^{* * * *}$ \\ * LSS \& LGEP, CNRS-SUPELEC, Gif-sur-Yvette 91192, \\ FRANCE, becherif@lss.supelec.fr \\ ** LCIS, ESISAR 50, rue Barthélémy de Laffémas, BP 54 \\ 26902 Valence Cedex 9, FRANCE, \\ Eduardo.Mendes@esisar.inpg.fr \\ *** LSS, CNRS-SUPELEC, Gif-sur-Yvette 91192, \\ FRANCE, ortega@lss.supelec.fr \\ **** IRI-UPC, 08028 Barcelona, Spain, riera@iri.upc.edu
}

\begin{abstract}
This paper deal with the control of an isolated doubly-fed induction generator, driven by a prime mover, whose stator is connected to the stator of an induction motor with an inertia flywheel. The rotor voltage of the generator is regulated by a bidirectional converter. The main interest of this configuration is that it permits a bidirectional power flow between the motor, which may operate in regenerative mode, and the generator. In a previous paper the authors proposed a passivity-based controller to regulate the motor mechanical speed and the rotor flux norm. Since this kind of controllers achieve stabilization via energy-balancing, regulation of the energy flow in the system can be naturally incorporated. In this work experimental results are presented. The performances of the proposed scheme and a classical PI controller are compared. Copyright (C)2005 IFAC.
\end{abstract}

Keywords: nonlinear control, induction machines, passive systems, nonlinear systems.

\section{INTRODUCTION}

This paper considers the isolated operation of a Doubly-Fed Induction Generator (DFIG) driven by a prime mover, with its stator connected to a load - which is in this case an Induction Motor (IM) with a flywheel inertia. Isolated generating units are economically attractive, hence increasingly popular, in the new era of deregulated markets. The possibility of a DFIG supplying an isolated load has been indicated in Vicatos and Tagopoulos (1989) and Bogalecka (1993) where

1 The authors would like to thank Enric Fossas and Miguel Allué from IRI-UPC, Barcelona, Spain for their hospitality and help in the practical implementation. some mention is made of the steady-state control problem. Mebarki and Lipczynsky (1995) propose a system in which the rotor is supplied from a battery via a PWM converter, and show experimental results from a $200 \mathrm{~W}$ prototype. A control system based on regulating the rms voltage of the DFIG is used, which results in large voltage deviations and very slow recovery following load changes. DFIGs have also been studied in Caratozzolo et al. (2002a,b), where feedback linearization and sliding mode principles are used for the design of motor speed controllers, and Peresada et al. (2003) where a controller based on field-oriented principles is proposed. In Battle et al. (2004) an Interconnection and Damping Assignment PBC is 
applied to a DFIG with a flywheel latched directly to the transmission line.

A Standard Passivity-Based Controller (SPBC) Ortega et al. (1998) was proposed in Becherif et al. (2003), where some stability analysis and simulations were given. Following the SPBC philosophy the whole system is decomposed as the feedback interconnection of passive mechanical and electrical subsystems. The electrical subsystem is controlled by an SPBC that regulates torque and rotor flux amplitude of the IM, while tracking of the mechanical rotor speed of the IM is ensured with a standard PI. As explained in Ortega et al. (1998) the SPBC is set up building a copy of the system that achieves asymptotic inversion, hence particular attention should be paid to the stable invertibility properties of the system.

In this paper we present the practical implementation of the SPBC on a $200 W$ prototype of a DFIG interconnected with an IM which is available in the Institute of Robotics and Industrial Informatics of the Polytechnic University of Catalonia. Real time control of the setup is done via RT-Linux. The experiments have shown that the SPBC ensures good performance and is robust with respect to machine parameters variations. A classical PI controller that regulates the stator currents was also experimentally tested and compared with the SPBC.

\section{SYSTEM CONFIGURATION AND MATHEMATICAL MODEL}

The configuration of the system considered in this paper is depicted in Fig. 1. It consists of a wound rotor DFIG, a squirrel cage IM and an external mechanical device that can supply or extract mechanical power. The IM and the DFIG are connected through their stator windings, while the rotor voltage of the DFIG is regulated by a bidirectional converter. The main interest of this configuration is that it permits a bidirectional power flow between the motor, which may operate in regenerative mode, and the generator.

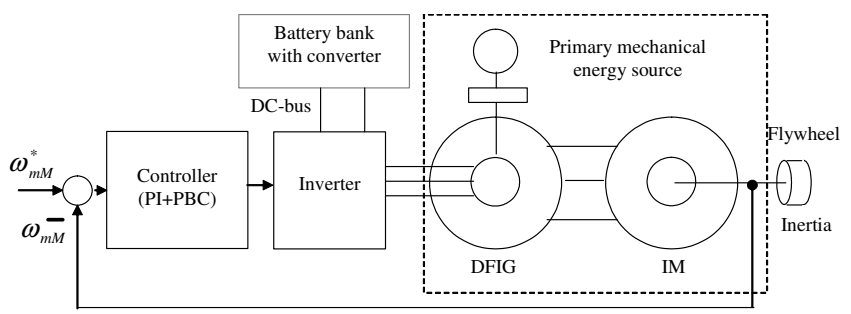

Fig. 1. System configuration with speed controller.

Fig. 2 shows a power port viewpoint description of the system. The DFIG is a three-port system with conjugated power port variables ${ }^{2}$ prime mover torque and speed, $\left(\tau_{L G}, \omega_{G}\right)$, and the rotor and stator voltages and currents, $\left(v_{r G}, i_{r G}\right),\left(v_{s}, i_{s G}\right)$, respectively. On the other hand, the IM is a two-port system with port variables motor load torque and speed, $\left(\tau_{L M}, \omega_{M}\right)$, and stator voltages and currents. The DFIG and the IM are coupled through the stator interconnection:

$$
\begin{gathered}
v_{s G}=v_{s M} \\
i_{s G}=-i_{s M}
\end{gathered}
$$

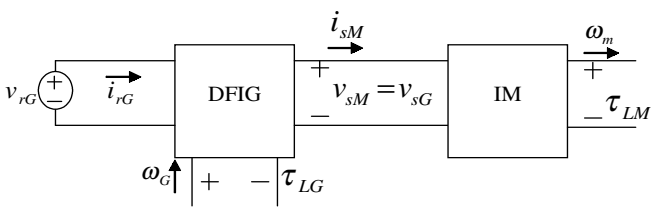

Fig. 2. Power port representation of the DFIG with IM.

To obtain the mathematical model of the overall system, ideal symmetrical phases with uniform air-gap and sinusoidally distributed phase windings are assumed. The permeability of the fully laminated cores is assumed to be infinite, and saturation, iron losses, end winding and slot effects are neglected. Only linear magnetic materials are considered. It is further assumed that all parameters are constant and known. Under these assumptions, the voltage balance equations for the machines are

$$
\begin{aligned}
\dot{\lambda}_{s G}+R_{s G} i_{s G} & =v_{s G} \\
\dot{\lambda}_{r G}+R_{r G} i_{r G} & =v_{r G} \\
\dot{\lambda}_{s M}+R_{s M} i_{s M} & =v_{s M} \\
\dot{\lambda}_{r M}+R_{r M} i_{r M} & =0
\end{aligned}
$$

where $\lambda_{s G}, \lambda_{r G}\left(\lambda_{s M}, \lambda_{r M}\right)$ are the two-dimensional stator and rotor fluxes of the DFIG (IM, resp.), $L_{s G}, L_{r G}, L_{m G}\left(L_{s M}, L_{r M}, L_{m M}\right)$ are the stator, rotor, and mutual inductances of the DFIG (IM, resp.); $R_{s G}, R_{r G}\left(R_{s M}, R_{r M}\right)$ are the stator and rotor resistances of the DFIG (IM, resp.).

The (power preserving) interconnection (1) induces an order reduction in the system. To eliminate the redundant coordinates, preserving the structure needed for application of SPBC, we define

$$
\lambda_{s G M}=\lambda_{s G}-\lambda_{s M}
$$

which upon replacement in (2), and after some simple manipulations, yields:

$$
\dot{\lambda}+R i=\left[\begin{array}{c}
I_{2} \\
0
\end{array}\right] v_{r G}
$$

\footnotetext{
2 The qualifier "conjugated power" is used to stress the fact that the product of the port variables has the units of power.
} 
where

$$
\lambda=\left[\begin{array}{c}
\lambda_{r G} \\
\lambda_{s G M} \\
\lambda_{r M}
\end{array}\right], \quad i=\left[\begin{array}{c}
i_{r G} \\
i_{s G} \\
i_{r M}
\end{array}\right]
$$

the resistance matrix is

$$
R=\operatorname{diag}\left\{R_{r G} I_{2},\left(R_{s G}+R_{s M}\right) I_{2}, R_{r M} I_{2}\right\} .
$$

To complete the model of the electrical subsystem, we recall the following relation between the fluxes and currents

$$
\lambda=L(\theta) i
$$

where the inductance matrix takes in this case the form

$L(\theta)=\left[\begin{array}{ccc}L_{r G} I_{2} & L_{m G} e^{-J n_{G} \theta_{G}} & 0 \\ L_{m G} e^{J n_{G} \theta_{G}} & \left(L_{s G}+L_{s M}\right) I_{2} & -L_{m M} e^{J n_{M} \theta_{M}} \\ 0 & -L_{m M} e^{-J n_{M} \theta_{M}} & L_{r M} I_{2}\end{array}\right]$

where $n_{G}, n_{M}$ denote the number of pole pairs, $\theta_{G}, \theta_{M}$ the mechanical rotor positions (with respect to the stator) and, to simplify the notation, it is introduced:

$$
\begin{gathered}
\theta=\left[\begin{array}{l}
\theta_{G} \\
\theta_{M}
\end{array}\right], \quad J=\left[\begin{array}{cc}
0 & -1 \\
1 & 0
\end{array}\right]=-J^{\top}, \\
e^{J x}=\left[\begin{array}{cc}
\cos (x) & -\sin (x) \\
\sin (x) & \cos (x)
\end{array}\right]=\left(e^{-J x}\right)^{\top} .
\end{gathered}
$$

It is recalled that, due to physical considerations, $R>0$ and $L(\theta)=L^{\top}(\theta)>0$.

A state-space model of the (6-th order) electrical subsystem is finally obtained replacing (4) in (3) as

$$
\Sigma_{e}: \dot{\lambda}+R L(\theta)^{-1} \lambda=\left[\begin{array}{c}
I_{2} \\
0
\end{array}\right] v_{r G}
$$

The mechanical dynamics is obtained from Newton's second law and is given by

$$
\Sigma_{m}: J_{m} \ddot{\theta}+B_{m} \dot{\theta}=\tau-\tau_{L}
$$

where $J_{m}=\operatorname{diag}\left\{J_{G}, J_{M}\right\}>0$ is the mechanical inertia matrix, $B_{m}=\operatorname{diag}\left\{B_{G}, B_{M}\right\} \geq 0$ contains the damping coefficients, $\tau_{L}=\left[\tau_{L G}, \tau_{L M}\right]^{\top}$ are the external torques, that are assumed constant in the sequel. The generated torques are calculated as usual from

$$
\tau=\left[\begin{array}{c}
\tau_{G} \\
\tau_{M}
\end{array}\right]=-\frac{1}{2} \frac{\partial}{\partial \theta} \lambda^{\top} L(\theta)^{-1} \lambda .
$$

From (4), this alternative expression is obtained

$$
\tau=\frac{1}{2} \frac{\partial}{\partial \theta} i^{\top} L(\theta) i
$$

For later reference we observe that

$$
\tau_{M}=\frac{n_{M}}{R_{r M}} \dot{\lambda}_{r M}^{\top} J \lambda_{r M}
$$

Remark 1. It has been shown in Battle et al. (2004) that the zero dynamics of the DFIG with respect to the output $i_{s G}$ is unstable. On the other hand, some preliminary calculations (see Becherif (2004)) of the DFIG coupled to the IM reveal that the zero dynamics with respect to the outputs $\tau_{r M}$ and $\left|\lambda_{r M}\right|$ is unstable in a neighborhood of the line

$$
\tau_{r M}=-\alpha \dot{\theta}_{M}\left|\lambda_{r M}\right|, \quad \alpha>0 .
$$

Since SPBC relies on the assumption of stable invertibility, we will operate the system away from this region.

Remark 2. An input-output representation of the electrical subsystem of the DFIG interconnected to the IM is presented in Fig. 3. It is easy (5) to show that the mapping $\left(v_{r G}, \tau_{L G},-\tau_{L M}\right) \mapsto$ ${ }^{5}\left(i_{r G},-\omega_{G}, \omega_{M}\right)$ is passive.

\section{PROPOSED SPBC}

We recall briefly here the SPBC presented in Becherif et al. (2003). As usual in SPBC of electromechanical systems it has the nested loop control configuration depicted in Fig. 3, with $C_{i l}$ the inner-loop torque tracking SPBC and $C_{o l}$ the outer-loop PI speed controller. The reader is referred to Ortega et al. (1998) for motivation and additional details on this control configuration.

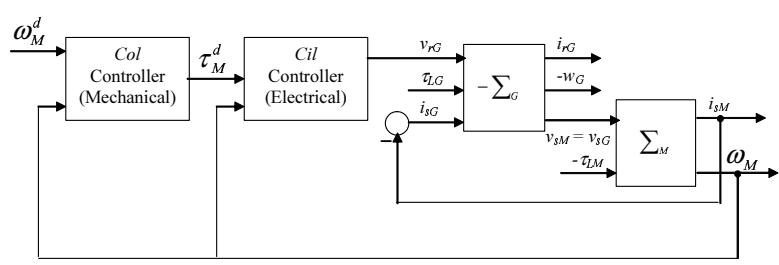

Fig. 3. Nested-loop control configuration.

To derive the torque tracking SPBC a "copy" of the electrical subsystem, but evaluated along some desired trajectories, is constructed as

$$
\begin{aligned}
{\left[\begin{array}{c}
I_{2} \\
0
\end{array}\right] v_{r G} } & =\dot{\lambda}^{d}+R L^{-1}(\theta) \lambda^{d} \\
\tau_{M}^{d} & =\frac{n_{M}}{R_{r M}}\left(\dot{\lambda}_{r M}^{d}\right)^{\top} J \lambda_{r M}^{d}
\end{aligned}
$$

where $\tau_{M}^{d}$ is the desired motor torque - to be generated by the speed PI loop-and $\lambda^{d}$ is a signal to be defined later and such that $\left|\lambda_{r M}^{d}\right|$ is constant and equal to some desired value. Compare with (6), (9). Equations (10) define the, so-called, implicit representation of the controller the explicit expression, which may be found in Becherif et al. (2003), is omitted for brevity.

Combining (10) with (6) yields the error equation for the fluxes 


$$
\dot{\tilde{\lambda}}+R L^{-1}(\theta) \tilde{\lambda}=0
$$

Consider now the storage function

$$
H_{\lambda}^{d}=\frac{1}{2} \tilde{\lambda}^{\top} R^{-1} \tilde{\lambda} \geq 0
$$

where $\tilde{\lambda}=\lambda-\lambda^{d}$.

The derivative of (12) along the trajectories of (11) yield

$$
\dot{H}_{\lambda}^{d}=-\tilde{\lambda}^{\top} L^{-1} \tilde{\lambda} \leq 0
$$

which ensures $\tilde{\lambda}(t) \rightarrow 0$, exponentially. The stability analysis is completed with some signal chasing and may be found in Becherif et al. (2003).

\section{EXPERIMENTAL VALIDATION}

In this section the proposed SPBC and a PI controller around stator currents are tested experimentally. The experimentations were done in a $200 W$ DFIG interconnected with an IM, whose parameters are shown in Table 1 . The setup is controlled using a computer working under RTLinux operating system, see Fig. 4.

The behavior of the system, under different load conditions and speed references, is tested applying a non-measured load torque to the IM. The latter is estimated using a speed observer based on IM rotor position measurement.

Since the asymptotic stability of the electrical subsystem $\Sigma_{e}$ is proven we can consider that in the steady state $\tau_{M} \rightarrow \tau_{M}^{d}$ (exponentially). Then,we have in the steady state the following:

$$
J_{M} \ddot{\theta}_{M}=\tau_{M}^{d}-\underbrace{\tau_{L M}-B_{M} \dot{\theta}_{M}}_{\tau_{M L}}
$$

Hence, a linear load torque observer is designed (with $l_{1}, l_{2}$ are design parameters):

$$
\begin{aligned}
\dot{\hat{\omega}}_{m M} & =\left(\tau_{M}^{d}-\hat{\tau}_{M L}\right) / J_{M}+l_{1}\left(\hat{\omega}_{m M}-\omega_{m M}\right. \\
\dot{\hat{\tau}}_{M L} & =l_{2}\left(\hat{\omega}_{m M}-\omega_{m M}\right)
\end{aligned}
$$

Table 1. Parameters of DFIG and IM

\begin{tabular}{|l||c|c|c|c|c|c|}
\hline & $R_{s}(\Omega)$ & $R_{r}(\Omega)$ & $L_{s}(\mathrm{mH})$ & $L_{r}(\mathrm{mH})$ & $L_{m}(\mathrm{mH})$ & $J\left(\mathrm{Nm}^{2} / \mathrm{rad}\right)$ \\
\hline \hline DFIG & 0.365 & 0.559 & 0.938 & 0.938 & 12.975 & $4.358 \times 10^{-3}$ \\
\hline IM & 0.5 & 0.2 & 1.2 & 1.2 & 9.00 & $1.1 \times 10^{-3}$ \\
\hline
\end{tabular}

\section{1 $S P B C$}

Figure 5 presents the response of the system to changes in the IM reference speed and load torque.
It has to be noticed that the IM rated torque is $0.7 \mathrm{Nm}$, hence the applied load torque is two times bigger than the nominal value.

The IM speed tracks very well the reference, i.e. low overshoot and no steady state error are observed. Also, the speed observer exhibits a very good tracking behavior.

Figure 6 shows the associated currents and their references over some time periods. Steady state errors are observed in the stator currents tracking, this is because the goal of the designed SPBC is to track the IM speed and to keep internal signals bounded.

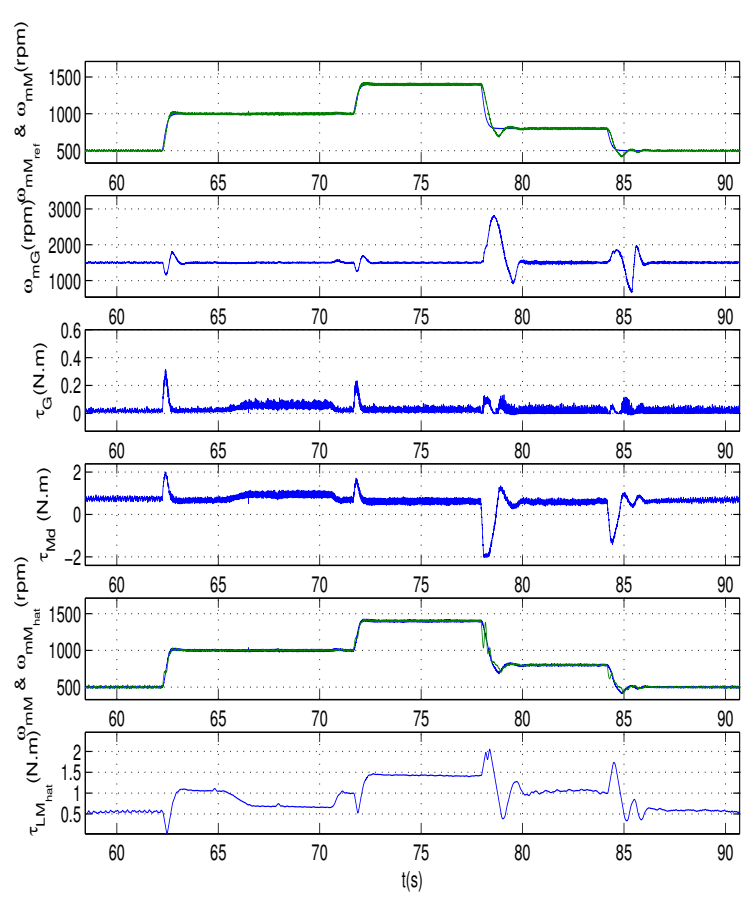

Fig. 5. (a) Motor speed and its reference. (b) Generator speed. (c) Generator torque. (d) Motor desired torque.(e) Real and estimated IM speed. (f) Estimated IM load torque.
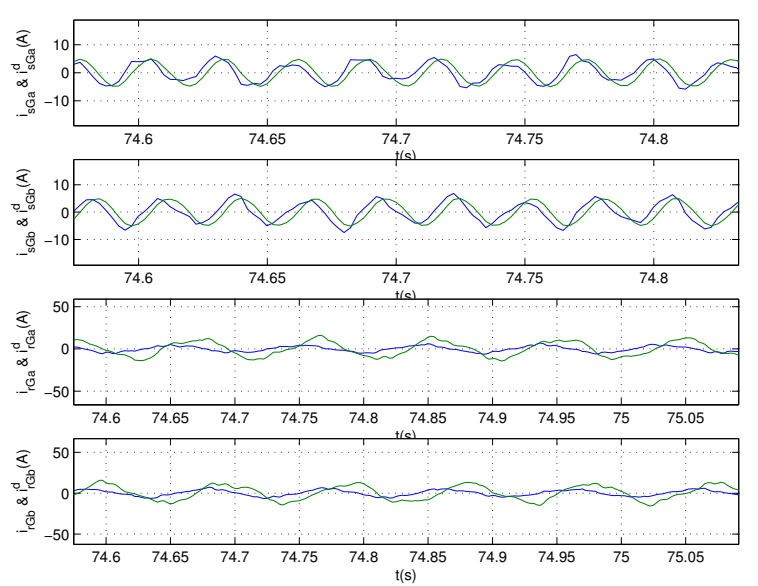

Fig. 6. (a) $i_{s G a}, i_{s G a}^{d}$. (b) $i_{s G b}, i_{s G b}^{d}$. (c) $i_{r G a}, i_{r G a}^{d}$. (d) $i_{r G b}, i_{r G b}^{d}$ 


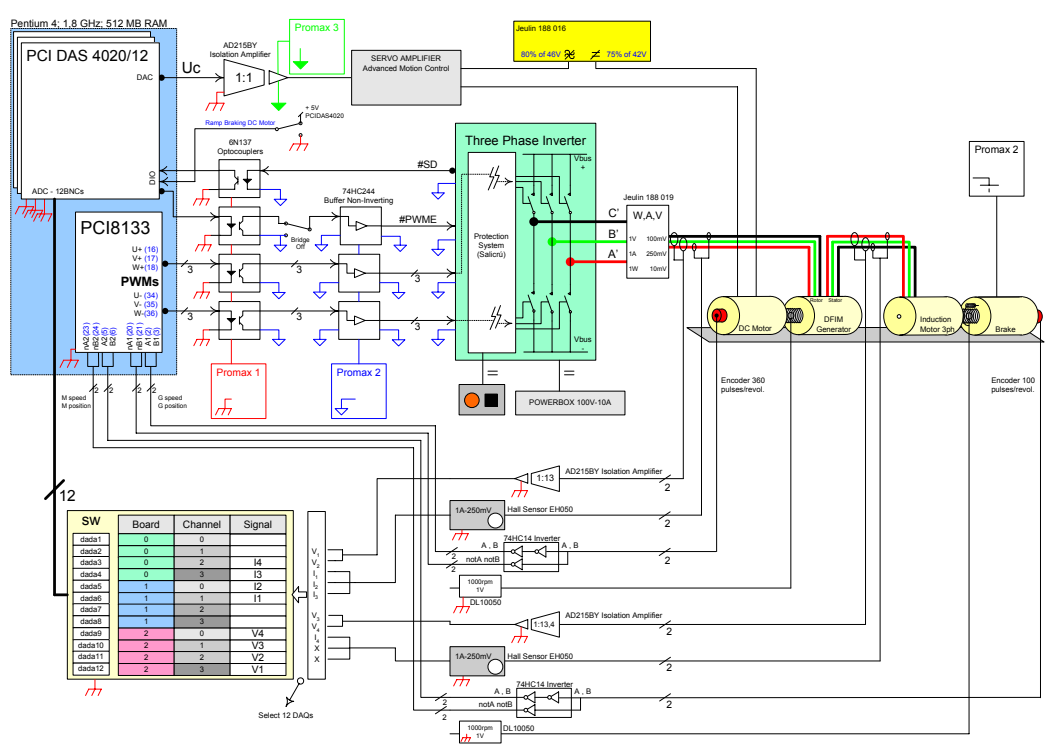

Fig. 4. Experimental setup

\subsection{PI control in currents}
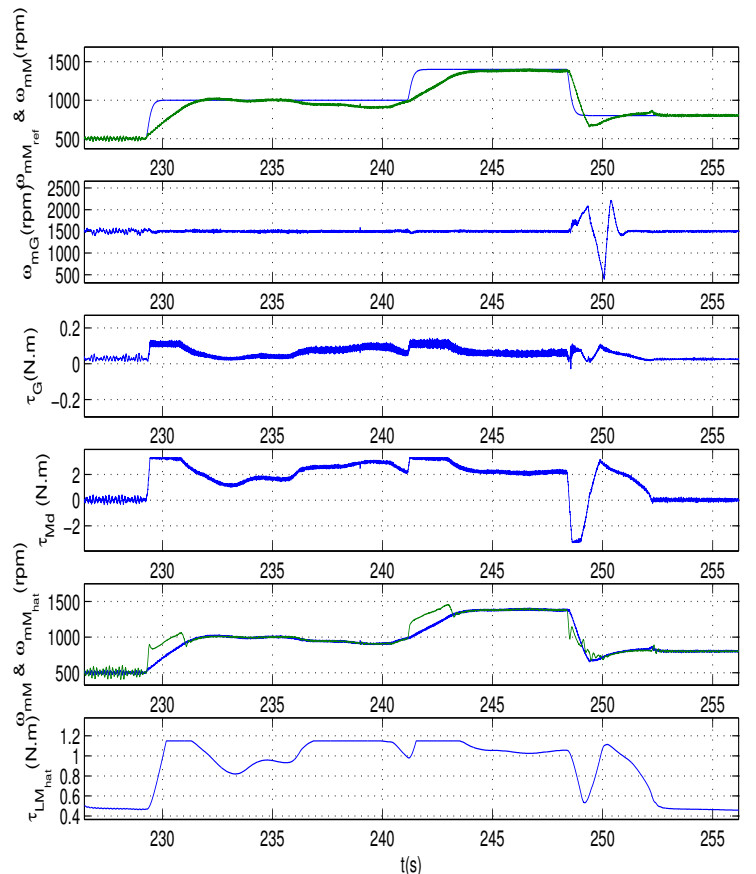

Fig. 7. (a) Regulated Motor speed and it's reference. (b) Generator speed.(c) Generator torque. (d) Motor desired torque.(e) $\omega_{m M}$, $\hat{\omega}_{m M}$. (f) $\hat{\tau}_{M L}$.

For each stator current a PI controller was designed. Figures 7 and 8 show the obtained results. It is clearly shown that the system behavior is much degraded in comparison with the SPBC. Even though there is no IM speed error in steady state, the speed does not track its reference during transients, and there is a speed error when a load torque is applied. This is mainly due to the saturation of the desired IM torque at a value four times its nominal value. Consequently, the
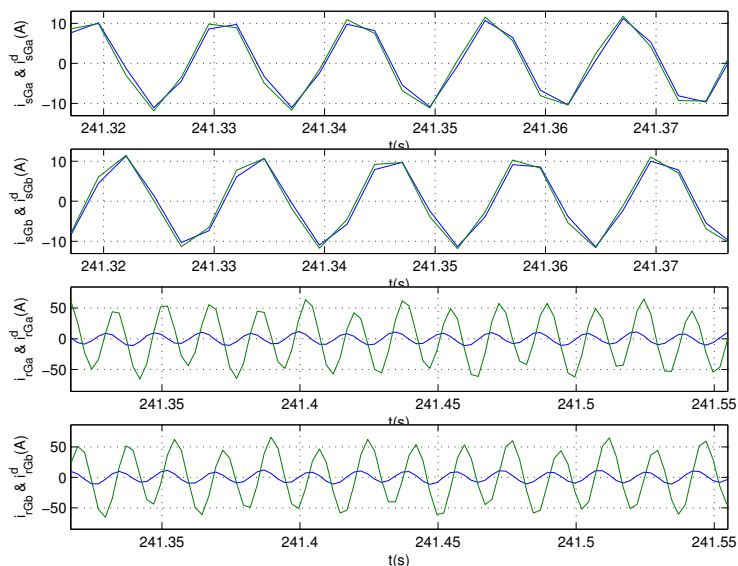

Fig. 8. (a) $i_{s G a}, i_{s G a}^{d}$. (b) $i_{s G b}, i_{s G b}^{d}$. (c) $i_{r G a}, i_{r G a}^{d}$. (d) $i_{r G b}, i_{r G b}^{d}$

stator currents are very large, i.e. their magnitude is about two times the one obtained with SPBC. Then, important stator losses can be expected.

These results show that PI control of the stator currents is not efficient for the control of the system. The SPBC shows much better behavior.

\subsection{Robustness tests}

In order to test the performance of the implemented controllers in the presence of parameter variations, changes in the DFIG and IM rotor and stator resistances are emulated. All the resistances of the two machines are suddenly decreased by $40 \%$ when the "Switch" signal value goes from 0 to 1 (see Figure 9).

Figure 9 presents the obtained results for the SPBC showing that it is quite insensitive to large change in machines resistance. Similar behavior may be observed for the PI in Figure 10. 

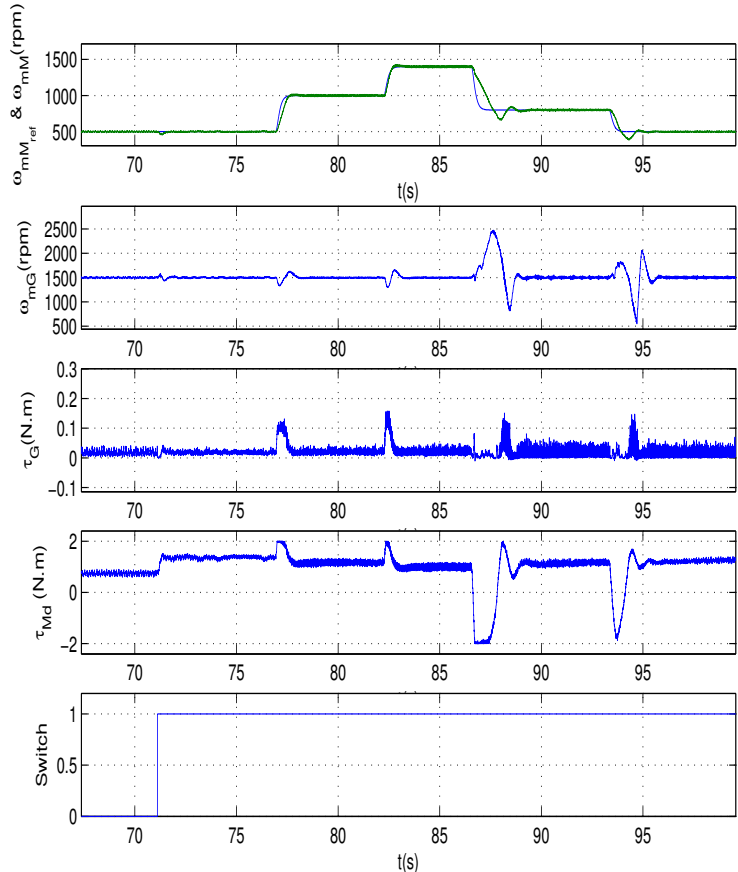

Fig. 9. (a) Regulated Motor speed and its reference. (b) Generator speed.(c) Generator torque. (d) Motor desired torque.(e) Switch.
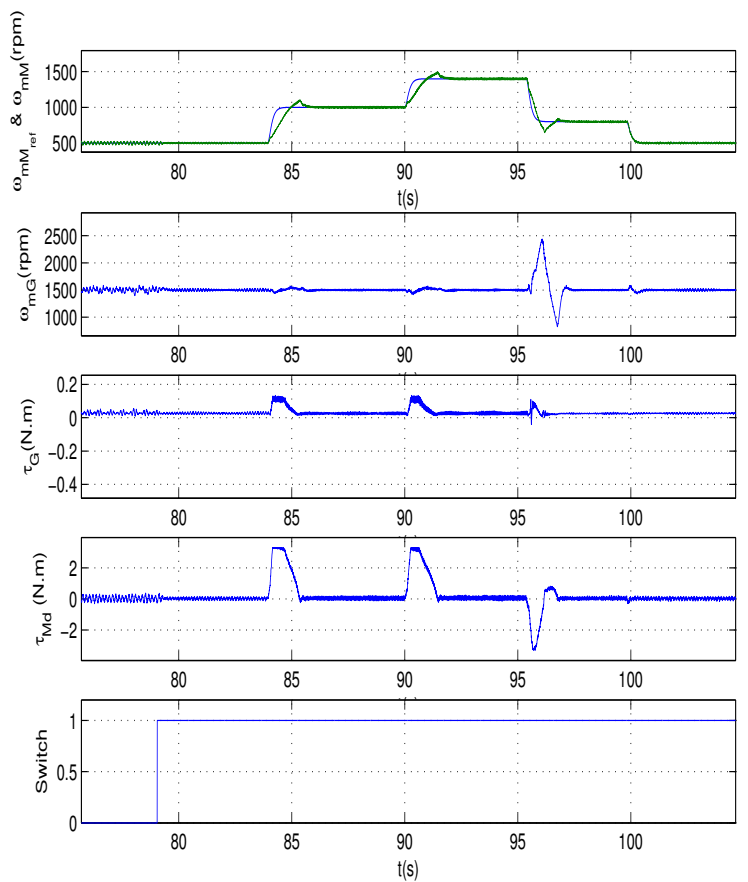

Fig. 10. (a) Regulated Motor speed and its reference. (b) Generator speed.(c) Generator torque. (d) Motor desired torque.(e) Switch.

\subsection{Quantitative comparison of SPBC and PI}

In Table 2 we compare the behavior of the proposed SPBC and PI algorithms, in the face of step changes in motor load torque, using the following performance indices (where $n$ is the length of the sampled data and $T$ is the sampling time):
- $e_{\omega_{M}}=\frac{1}{n T} \sum_{i=1}^{n}\left[\omega_{M}(i)-\omega_{R e f M}(i)\right]^{2}$;

- $e_{i_{s G a}}=\frac{1}{n T} \sum_{i=1}^{n}\left[i_{s G a}(i)-i_{s G a}^{d}(i)\right]^{2}$

- Peak magnitude of $i_{s G a}$;

- $P_{\mathrm{avg}_{G}}=\frac{1}{n} \sum_{i=1}^{n}\left[\tau_{G}(i) \omega_{G}(i)\right]$, e.g., average instantaneous power absorbed by the DFIG;

- $P_{\operatorname{avg}_{M}}=\frac{1}{n} \sum_{i=1}^{n}\left[\tau_{M}(i) \omega_{M}(i)\right]$, e.g., average instantaneous absorbed power by the IM.

Table 2Comparison table of experimental results

\begin{tabular}{|c||c|c|}
\hline & SPBC & PI \\
\hline \hline$\tau_{L M}[\mathrm{~N} . \mathrm{m}]$ & $0.5 \rightarrow 1.45 \rightarrow 0.5$ & $0.5 \rightarrow 1.15 \rightarrow 0.5$ \\
\hline $\begin{array}{c}\text { settling time } \\
\text { of } \omega_{R e f M}\end{array}$ & $0.4 s$ & $0.4 s$ \\
\hline $\begin{array}{c}\text { settling time } \\
\text { of } \omega_{M}\end{array}$ & $0.1 s$ & $2 s$ \\
\hline$\omega_{G}[\mathrm{rpm}]$ & 1500 & 1500 \\
\hline$e_{\omega_{M}} \times 10^{5}$ & 2.9 & 38.6 \\
\hline$e_{i_{s G a}} \times 10^{3}$ & 3.7 & 0.25 \\
\hline $\begin{array}{c}\text { Magnitude } \\
\text { of } i_{s G a}[\mathrm{~A}] \simeq\end{array}$ & 5 & 10 \\
\hline$P_{\mathrm{avg}_{G}}[\mathrm{~W}]$ & 4.7 & 9 \\
\hline$P_{\mathrm{avg}_{M}}[\mathrm{~W}]$ & 58.9 & 177.9 \\
\hline
\end{tabular}

Even though the load changes are smaller for the PI, the SPBC exhibits far better behavior in all, but the current tracking, indices.

\section{REFERENCES}

C. Battle, A. Doria, and R. Ortega. Power flow control of a doubly-fed induction machine coupled to a flywheel. Proc. IEEE Conf. Control Appl., CCA'2004, Taipei, Taiwan., September 2-4 2004.

M. Becherif. Contribution aux techniques de façonnement d'énegie : Application à la commande des systèmes électromécaniques. Phd thesis, Université Paris Sud XI, France, 2004.

M. Becherif, R. Ortega, E. Mendes, and S. Lee. Passivitybased control of a doubly-fed induction generator interconnected with an induction motor. $42^{\text {nd }}$ Conf. on Decision and Control, pp:5657-5662, 2003.

F. Bogalecka. Dynamics of the power control of a double fed induction generator connected to the soft power grid. ISIE Intern. Symp. on Indust. Electr., pp:509513, 1993.

P. Caratozzolo, E. Fossas, and J. Riera. Nonlinear control of an isolated motion system with DFIG. 15th IFAC World Congress on Automatic Control, Barcelone, Espagne, 2002a.

P. Caratozzolo, E. Fossas, and J. Riera. Robust nonlinear control of an isolated motion system. CIEP Intern. Power Electr. Congress, 2002b.

A. Mebarki and R.T. Lipczynsky. Novel variable speed constant frequency generation system with voltage regualtion. EPE Europ. Conf. on Power Electr. and Appl., 2: 465-471, 1995.

R. Ortega, A. Loria, P.J. Nicklasson, and H. SiraRamirez. Passivity-based control of Euler-Lagrange systems. Springer-Verlag, Berlin, 1998.

S. Peresada, A. Tili, and A. Tonielli. Indirect stator flux-oriented output feedback control of a doubly-fed induction machine. IEEE Trans. Control Systems Technology, 11(6), 2003.

M.S. Vicatos and J.A. Tagopoulos. Steady state analysis of a doubly-fed induction generator under sychronous operation. IEEE Trans. on Energy Conversion, 4(3): 495-501, 1989. 